\title{
LAS REDES SOCIALES Y SU IMPACTO PUBLICITARIO EN ECUADOR (2020)
}

\author{
AUTORES: $\quad$ Klevers Alexander Mendez Pico ${ }^{1}$ \\ Kirenia Maldonado Zuñiga ${ }^{2}$
}

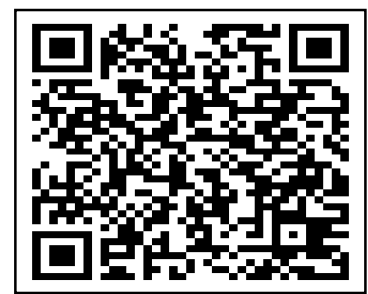

DIRECCIÓN PARA CORRESPONDENCIA: mendez-klevers9331@unesum.edu.ec

Fecha de recepción: 12/01/2021

Fecha de aceptación: 25/06/2021

\section{RESUMEN}

Desde la primera red social, SixDegrees que se creó en 1997, hasta el presente fecha , la evolución de la comunicación tecnológica ha sufrido una gran cantidad cambios para bien y de mejoras, que han impulsado a mercados tan importantes que, en el siglo pasado, hubieran fracasado rotundamente ,aunque parezca mentira, las redes sociales ha generado un impulso , a la industria publicitaria siendo este uno de los aspectos, más explotados por las empresas y compañías que busca cuñas publicitarias actualmente en Ecuador , compañías e instituciones ven esta herramienta tecnológica como un lecho para potencializar a través de la publicidad una idea, producto servicio o imagen .En el desarrollo de la investigación destaco el método histórico lógico, importante para la recopilación de información , gracias a este método se pudo constatar que actualmente las redes sociales además de ser un medio de comunicación las redes sociales ayudan a usuarios profesionales que desean crear grandes proyectos tecnológicos y que este tenga un mayor alcance en el país dejando atrás la época de gráficos pixelados e imágenes publicitando un producto de manera convencional , todo esto gracias a la evolución tecnológica de esta herramienta tecnológica. En el apartado laboral le da empresa, el plus de tener una ventaja considerable frete a otras compañías que no posean este componente esencial. Por estas razones está considerado que las redes sociales en Ecuador son realmente muy importantes y tiene un potencial impacto en este siglo digital, ya que abarcan una gran cantidad de usuarios, calculándose que el país 13.8 millones de usuarios frecuentan diariamente una red social por lo que se puede asegurar que, en un breve lapso, toda la industria publicitaria adoptara las redes sociales como principal medio publicitario.

PALABRAS CLAVE: comunicación; empresa; mercado.

\section{SOCIAL MEDIA AND THEIR IMPACT IN ECUADOR SUMMARY}

From the first social network, SixDegrees that was created in 1997, to the present date, the evolution of technological communication has undergone a great number of changes for the

1Estudiante de la carrera de Tecnología de la Información en la Universidad Estatal del sur de Manabí JipijapaManabí-Ecuador.

2Máster en Ciencias de la Educación, Licenciada en Educación Informática. Docente de la carrera en Ingeniería en Tecnologías de la Información. Universidad Estatal del Sur de Manabí. Jipijapa, Manabí, Ecuador. 
better and improvements, which have driven such important markets that, in the last century, would have completely failed, oddly enough, social networks have generated a boost, to the advertising industry, this being one of the aspects, most exploited by companies and companies that are currently looking for advertising spots in Ecuador, companies and institutions see this technological tool as a bed to potentiate an idea, product, service or image through advertising. In the development of the research I emphasize the logical historical method, important for the collection of information, thanks to this method it was found that currently social networks in addition to being a means of communication social networks help professional users who want to create large projects technological ects and that it has a greater reach in the country leaving behind the era of pixelated graphics and images advertising a product in a conventional way, all this thanks to the technological evolution of this technological tool. In the labor section, it gives you a company, the plus of having a considerable advantage over other companies that do not have this essential component. For these reasons, it is considered that social networks in Ecuador are really very important and have a potential impact in this digital century, since they cover a large number of users, calculating that the country 13.8 million users daily frequent a social network, therefore It can be assured that, in a short period of time, the entire advertising industry will adopt social networks as the main advertising medium.

KEY WORDS: communication; company; market.

\section{INTRODUCCIÓN}

En la presente investigación se tocarán temas relacionados a manejo de redes sociales como medio publicitario y el cambio que ha provocado en el comportamiento de las personas. Lo que al principio generaba algo de temor en la privacidad, con el tiempo, ese temor se está perdiendo. Las personas se exponen mucho más en los medios sociales y esto es una oportunidad muy grande para el acercamiento de las empresas hacia ellos.

Por medio de las redes sociales, las compañías y empresas pueden conocer muchos datos de los usuarios para su beneficio. Entre la información obtenida esta la ubicación, estilo de vida, gustos, intereses y varios datos que desde un punto de vista estratégico se manejan para explotar al máximo el márquetin de una marca, organización o persona.

El uso de las redes sociales actualmente es una de las mejores herramientas para alcanzar los objetivos que se proponga la empresa, y no solo abarca el campo empresarial, también se utiliza la publicidad en estos medios al momento de querer fortalecer una marca o hacer público un nuevo producto en la población ecuatoriana, dentro de los periodos electorales los medios digitales promocionan diversos partidos políticos llegando a ganar más seguidores o adeptos.

La velocidad promedio de navegación en la población ecuatoriana es de 3.4 megabytes por segundo, algo realmente bajo comparado con países de la región. Pero esto no ha sido un impedimento, según datos de (Ponce, 2020) el consumo de redes sociales por parte de ecuatorianos en enero del año 2020 es de 13.8 millones de usuarios, repartidos en Facebook 12.04 millones de usuarios conectados, Instagram 4.02 millones, LinkedIn 2.587 millones, Tiktok 1.4 millones, Twitter 0.9 millones y Pinterest con 880.000 usuarios.

En torno a lo planteado anteriormente, el tema de las redes sociales y la publicidad que en ella se puede encontrar, es tema de interés en esta investigación, razón por la cual, en el presente artículo a través de una revisión sistemática de diferentes documentos y publicaciones, se plantea determinar cuál es el impacto que tienen estos medios de comunicación al ser utilizados como plataformas publicitarias en el Ecuador.

60 UNESUM-Ciencias. Publicación cuatrimestral. Vol. 5, Año 2021, No. 4 (Número Especial) 


\section{DESARROLLO}

Durante los últimos años el uso de las redes sociales en Ecuador ha tenido una gran acogida, cerca del $70 \%$ de los usuarios de internet son partícipes de alguna red social. Las redes sociales a nivel mundial son variadas y tienen diferentes plataformas dirigidas a móviles y pagina web con diferentes enfoques que se dirigen a determinado público.

El éxito proviene por la innovación y las ventajas que brindan o las nuevas formas de comunicarse con los demás. Pero la implementación de diferentes tecnologías dentro de las redes sociales, convierten actualmente a una red social en un buen medio para a publicidad y más aún si se tiene una gran idea publicitaria que podría generar un impacto y fortalecer un negocio, idea, figura pública, pensamientos, lanzamientos tecnológicos, prestamos de algún servicio o influir en las nuevas tendencias del país.

Gracias a la era digital en la que vivimos, la publicidad ha expandido sus horizontes hacia el mundo tecnológico y le ofrece mayor visibilidad a un usuario. Actualmente las empresas invierten en más anuncios por internet debido a que los anuncios en redes sociales se convirtieron según (Villacís, 2018), en medios de comunicación donde se pueden proponer conducir una idea o mensaje sobre alguna marca o tema, brindando la oportunidad de atraer a nuevos consumidores, a través de nuevas herramientas que se adaptan a las empresas, presupuestos y a las necesidades.

Además, explica que estas herramientas, implementaron propagandas virtuales hace más de 10 años. Gina Macías, máster en Finanzas, dice que las redes sociales son efectivas con un buen manejo e indica que "Las redes sociales son consideradas hoy en día un canal más de comunicación con los clientes o potenciales clientes y ya no son a elección para un emprendimiento o un negocio; son una necesidad porque nos permiten estar conectados con nuestra audiencia. Hay que tener una estrategia y saber a quién llegar con nuestro mensaje y qué vamos a comunicar".

Por otro lado, Paola Aulestia expresa que, "En la región Costa, la red social que tiene mayor impulso es Instagram para dar a conocer sus productos o servicios, en el caso de la región Sierra, la consideración ha sido Facebook, pero está tomando un ligero impulso Instagram.

$\mathrm{Si}$ es una persona independiente es necesario que tenga presencial en LinkedIn, entonces las estrategias cambian de acuerdo con el segmento del mercado el cual yo me enfoco y a la actividad que realiza"(Stephanie Gómez, 2020).

En Ecuador existen aproximadamente 12 millones de habitantes lo que tienen internet, representando que un $70 \%$ de la población tiene acceso al servicio, las mismas cifras son las que representan el total de usuarios de redes sociales y la cantidad de la población que acceso a las misma, casi la totalidad de los ecuatorianos tiene una línea telefónica, y todos los usuarios de internet, son usuarios de las redes sociales.

El promedio de uso de una red social promedio es de 3 a 12 minutos aproximadamente, comenzando con el buscador más famoso del mundo, Google, que en promedio recibe 14.6 visitas de 12 minutos con nueve segundos cada una. En este conteo también se encuentra YouTube, Facebook, Wikipedia, el banco de Pichincha, Netflix, mientras que para cerrar esta lista se encuentra MSN quien en promedio recibe 3.2 visitas de unos tres minutos y 23 segundos cada una.

FACEBOOK

(c) Universidad Estatal del Sur de Manabí. Jipijapa, Ecuador. 
Tiene una audiencia que abarca la mayor concentración de usuario en Ecuador, diariamente 12 millones de ecuatoriano visitan esta plataforma consumiendo videos recibiendo o enviando mensajes de texto y de diferentes maneras recibiendo publicidad de varias maneras.

\section{INSTAGRAM}

La cantidad de usuarios que pueden ser alcanzados por publicidades de organizaciones o emprendedores dentro de esta plataforma, es de un total de 3.90 millones de usuarios lo que representa un $29 \%$ de la población con un publicidad especialmente dirigida y orientada ligeramente hacia las mujeres.

\section{SNAPCHAT}

En promedio la cantidad de usuarios que visitan a diario esta plataforma es de 1.75 millones de usuarios, lo que comparado con la población total representa un $13 \%$.

En Twitter la cantidad de ecuatorianos que a diario consumen contenido es de 1.11 millones de usuarios, lo que comparado con el total de la población representa un 8.3\% de la misma.

\section{LINKEDIN}

Esta red social es utilizada por los profesionales para presentar ante los demás su síntesis curricular, una plataforma que cuenta con 2.40 millones de usuarios, dato que representa el $20 \%$ de la población (Xie, 2020).

\section{INTERNET COMO MEDIO PUBLICITARIO}

El internet se ha convertido en un medio publicitario para las pequeñas y grandes empresas , donde es posible a través de estos medios e entregar a los clientes reales y potenciales una publicidad con valor añadido, generar interacción entre los que pertenecen a las redes sociales o a aquellos que usan las herramientas de creación de anuncios publicitarios para la promoción de productos y servicios(Martin, 2011).

\section{COMERCIO ELECTRÓNICO EN ECUADOR}

El E-Commerce o comercio electrónico se encuentra en la mayoría de las redes sociales incluso, Facebook tiene un apartado especial para la compra y venta de productos. El marketing por internet se puede hacer a través de diferentes redes sociales, siendo que la parte inicial para poder establecer una buena comunicación entre clientes y empresas o emprendimientos es el desarrollo del e-Commerce, que en Ecuador es la "actividad económica basada en el ofrecimiento de productos o servicios para la compra o la venta, a través de medios digitales como internet.

Además, indica que, en ecuador a la publicidad en medios digitales, no está completamente estudiada ni existen cifras verificables. Aunque El consultor de mercados, Daniel Arévalo, sostiene que en el país la publicidad digital crece a través de tres frentes: Google, Facebook y aplicaciones para 'smartphones.

Según , (Ortega, 2019)indica que las redes sociales tienen un limitante para bien o para mal : la mayoría de los usuarios de Facebook y de las redes sociales oscila entre 16 y 25 años; los cuales son jóvenes que no invierten, no deciden, influyen pero indirectamente, no tienen mayor capacidad adquisitiva, solo consumen publicidad pero no generan ingresos a empresas e industrias

\section{PUBLICIDAD POLÍTICA EN REDES SOCIALES}

Es tan grande el impacto que se puede obtener al contratar un espacio publicitario en una red social en Ecuador que diferentes organismos controladores del país modificaron los reglamento para los diferentes partidos políticos

62 UNESUM-Ciencias. Publicación cuatrimestral. Vol. 5, Año 2021, No. 4 (Número Especial) 
La presidenta del organismo electoral, Diana Atamaint, aprobó en el año 2020 nuevos el reglamento que incluye a las redes sociales. Afirmó que en su conjunto el documento busca verificar el monto, origen, uso y destino de los recursos en las campañas electorales y así evitar casos de explotación y de que algún partido político invierta cantidades exorbitantes en cualquier medio publicitario en internet y sacar ventajas frente a los demás competidores.

El analista político Esteban Ron explicó que las novedades de este reglamento tienen su naturaleza en las reformas al Código de la Democracia, en cuanto a la aceptación de los medios digitales como parte de la promoción electoral (Jmmy, 2020).

METODOS UTILIZADOS

Método Analítico

Gracias a este método se pudo realizar el análisis correspondiente de manera objetiva y sistemática de las diferentes redes sociales que más presentan publicidad en Ecuador.

Método Bibliográfico

Con este método se recopilo la información, redactada por varios autores en obras literarias y revistas científicas, que sirvieron para conformar desarrollo teórico de este documento.

Método Histórico-lógico

Con ayuda de este método se logró realizar una búsqueda de información e indagar en los antecedentes de la investigación realizada.

\section{RESULTADOS}

Al culminar la investigación se procedió a realizar un análisis referente a la información obtenida, en donde se determinó que Facebook es la red social más frecuentada actualmente con más de 12 millones de ecuatorianos, además se puede apreciar que esta y otras redes sociales son frecuentada en la mayoría por usuarios móviles.

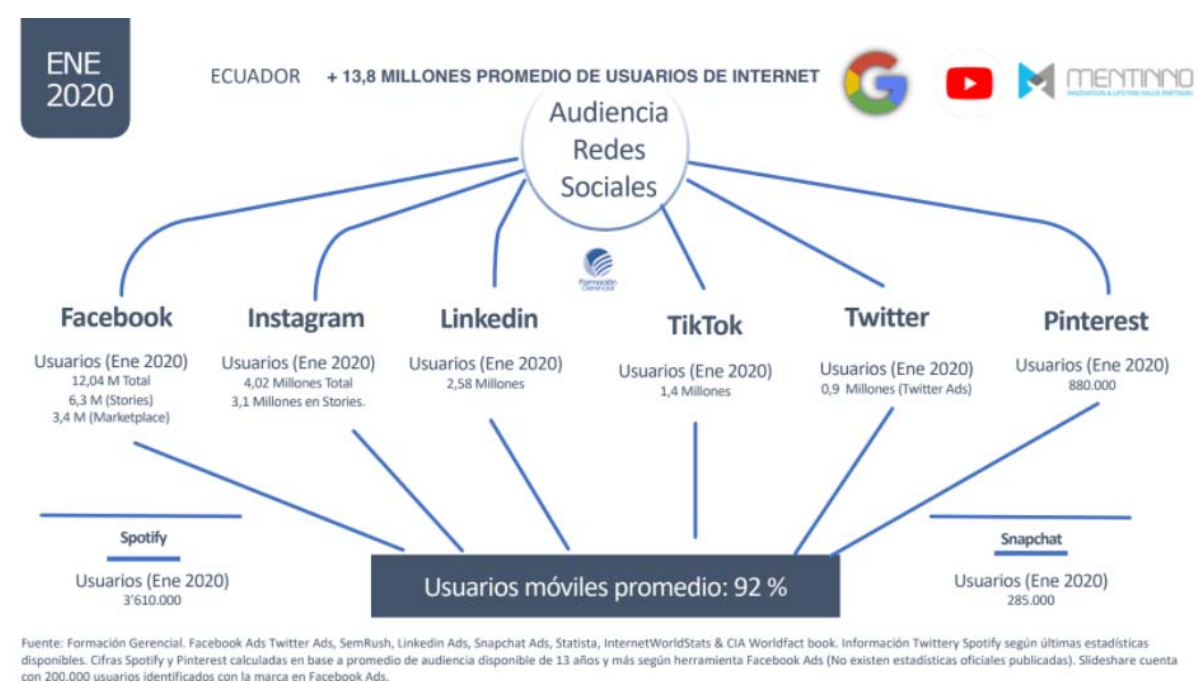

Figura 1:Frecuencia de usuarios en las redes sociales (Ponce , J, 2020). 
Guayaquil y Quito, destaca como las ciudades con mayor cantidad de usuarios que consumen contenido en redes sociales, por consiguiente, Guayaquil y Quito son las ciudades del Ecuador donde las empresas prefieren difundir mayor cantidad de publicidad.

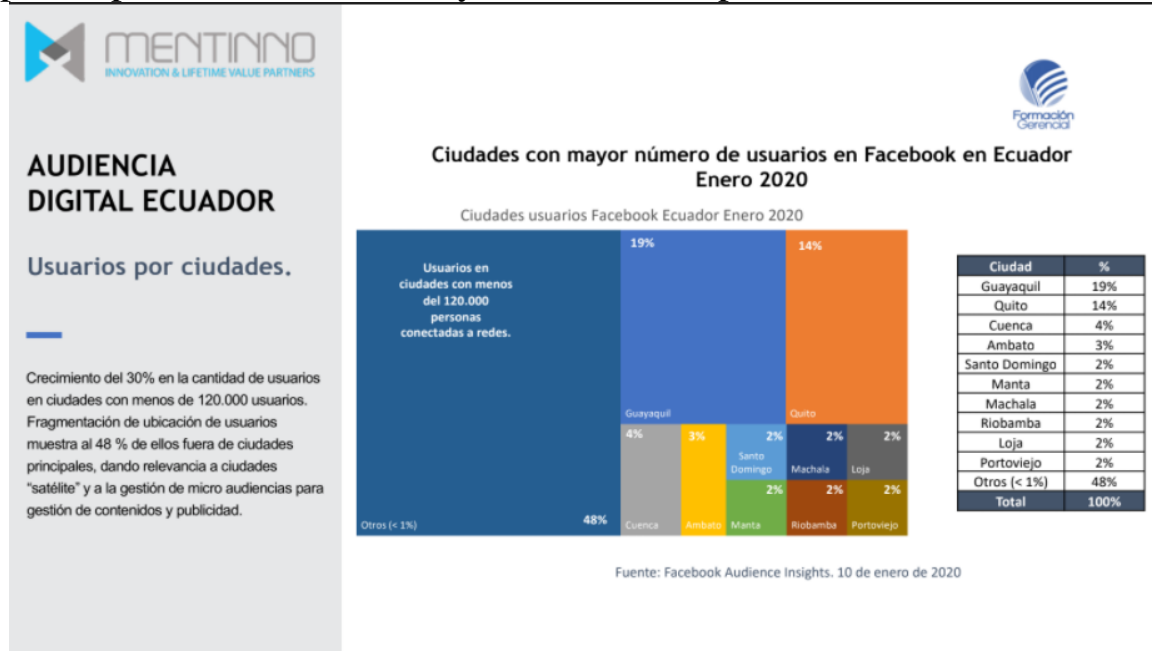

Figura 2: Difusión de información mediante las redes sociales (Ponce , J, 2020).

Para finalizar dentro de la inversión empresarial de publicidad en redes sociales destaca la participación de KAYAK y KIA Motor con la alarmante cifra de 280.8881 .852 millones de impresiones y 119.314.972 respectivamente

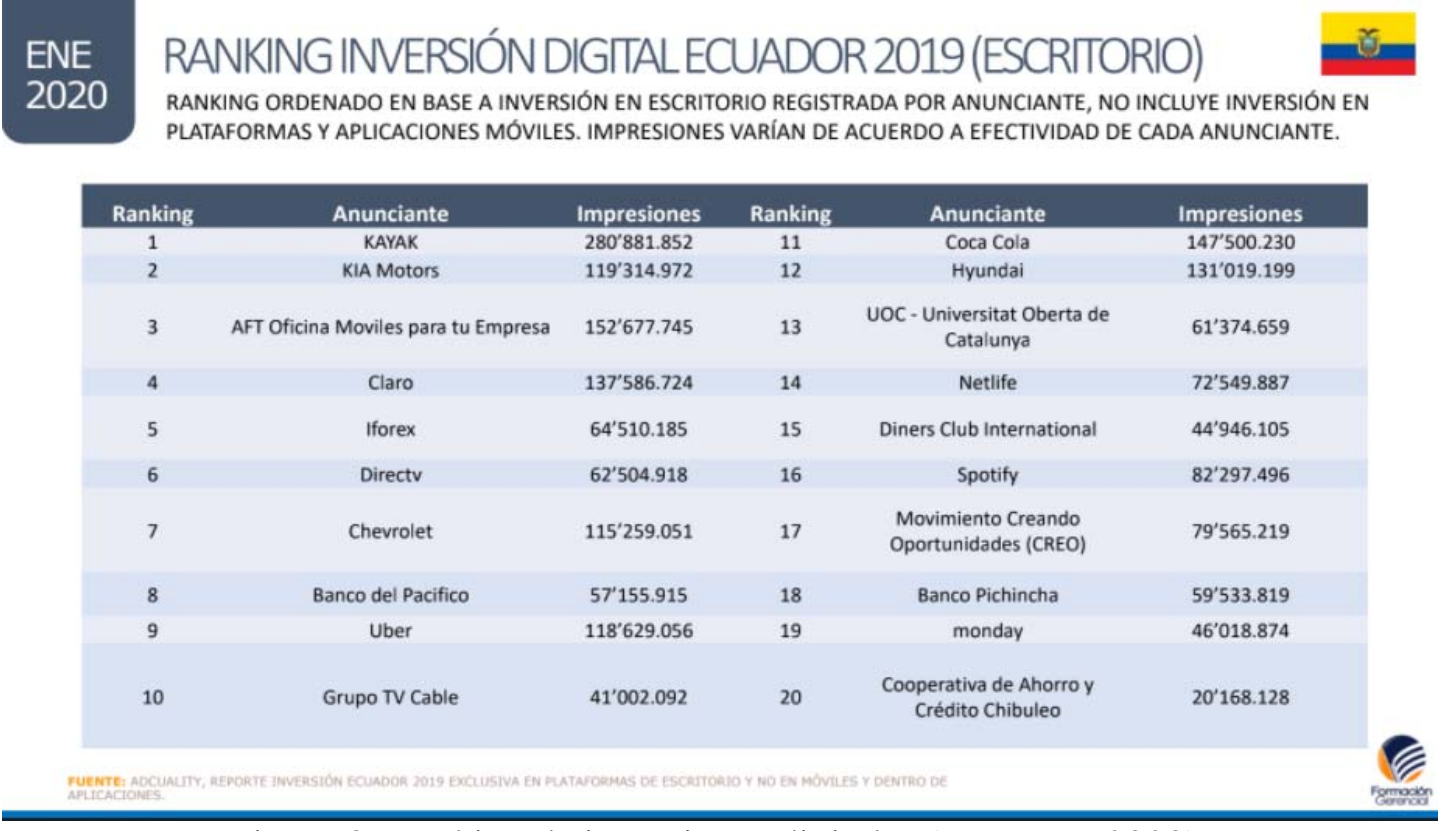

Figura 3: Ranking de inversiones digitales (Ponce , J, 2020).

El costo por cada mil impresiones es de 2.88 dólares ecuatorianos, demostrando el interés activo de las empresas por pactar publicidad en Redes Sociales gracias al fuerte impacto que en Ecuador tiene la publicidad en Redes.

64 UNESUM-Ciencias. Publicación cuatrimestral. Vol. 5, Año 2021, No. 4 (Número Especial) 


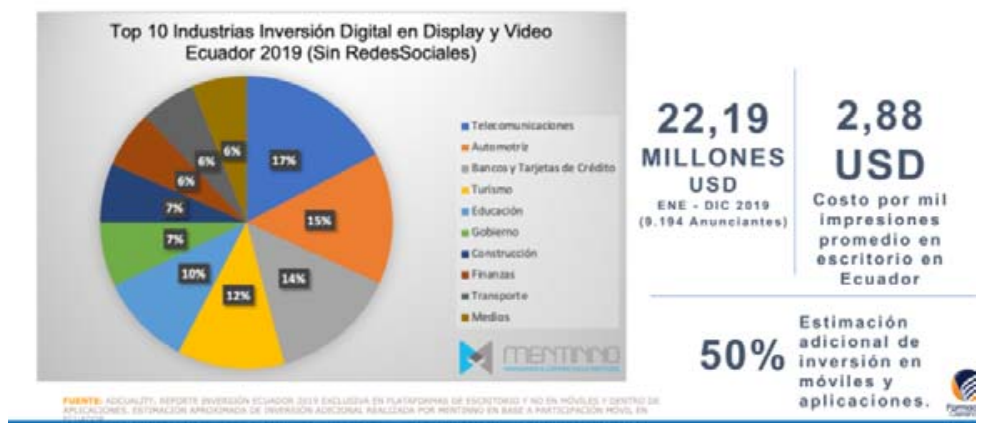

Figura 4: Inversiones públicas (Ponce , J, 2020).

\section{CONCLUSIONES}

Se presentó el siguiente articulo con el fin de facilitar al lector información clara y concisa, del impacto que tienen las redes sociales como medio publicitario en Ecuador, luego de realizar las investigaciones correspondientes se obtuvo un resultado positivo que demostró el gran impacto que tienen las redes sociales en actualidad, como medio publicitario por el uso constante de los ecuatorianos del internet específicamente en redes sociales como Facebook. Este fenómeno a generado, una migración de empresas publicitarias para tener un mayor alcance en campañas publicitarias, dado a la alta cantidad de usuarios que consumen contenido en las redes sociales. Además, quedo demostrado que la mayor parte de las personas que consumen contenido en redes sociales son adolescentes que no superan los 25 años, y aunque sea un buen medio publicitario al contener la mayoría de los usuarios gente de corta edad, con un poder adquisitivo inferior es posible que las ventas no sean las esperadas.

\section{REFERENCIAS BIBLIOGRÁFICAS}

Jmmy, G. (2020). Las redes sociales deben dar informe de la publicidad política | El Comercio. Opgehaal 21 Januarie 2021, van https://www.elcomercio.com/actualidad/redes-sociales-informe-publicidad-politica.html

Martin, P. (2011). Vista de Las redes sociales y herramientas de publicación de anuncios digitales para mejorar la promoción de servicios arquitectónicos en Loja | INNOVA Research Journal. Opgehaal 21 Januarie 2021, van https://revistas.uide.edu.ec/index.php/innova/article/view/751/1154

Ortega, F. (2019). Ecuador tiene un mercado publicitario con multiplicidad de actores | Revista Líderes. Opgehaal 21 Januarie 2021, van https://www.revistalideres.ec/lideres/ecuador-mercado-publicitario-multiplicidadactores.html

Ponce , J, C. (2020). Ecuador Estado Digital Ene 2020 F2.pdf - Google Drive. Opgehaal 21 Januarie 2021, van https://drive.google.com/file/d/1Yd5h5z2xXyABzj05-j6DEtVhS2O5Ywmw/view

Jmmy, G. (2020). Las redes sociales deben dar informe de la publicidad política | El Comercio. Opgehaal 21 Januarie 2021, van https://www.elcomercio.com/actualidad/redes-sociales-informe-publicidad-politica.html

Martin, P. (2011). Vista de Las redes sociales y herramientas de publicación de anuncios digitales para mejorar la promoción de servicios arquitectónicos en Loja | INNOVA Research Journal. Opgehaal 21 Januarie 2021, van https://revistas.uide.edu.ec/index.php/innova/article/view/751/1154

Ortega, F. (2019). Ecuador tiene un mercado publicitario con multiplicidad de actores | Revista Líderes. Opgehaal 21 Januarie 2021, van https://www.revistalideres.ec/lideres/ecuador-mercado-publicitario-multiplicidad- 
actores.html

Ponce , J, C. (2020). Ecuador Estado Digital Ene 2020 F2.pdf - Google Drive. Opgehaal 21 Januarie 2021, van https://drive.google.com/file/d/1Yd5h5z2xXyABzj05-j6DEtVhS2O5Ywmw/view

66 UNESUM-Ciencias. Publicación cuatrimestral. Vol. 5, Año 2021, No. 4 (Número Especial) 